\title{
Timed up and go test predicts mortality in older adults in Peru: a population-based cohort study
}

\author{
Edson J. Ascencio', Gustavo D. Cieza-Gómez' ${ }^{1}$ Rodrigo M. Carrillo-Larco ${ }^{2,3}$ and Pedro J. Ortiz ${ }^{14^{*}}$
}

\begin{abstract}
Background: While there is evidence about stablished risk factors (e.g., raised blood pressure) and higher mortality risk in older population, less has been explored about other functional parameters like the Timed Up and Go test and the Gait Speed in older people at low- and middle-income countries. We aimed to study these mobility tests as predictors of mortality in a population of older people in Peru.

Methods: Population-based prospective cohort study (2013-2020). Random sampling of people aged 60+ years in a community of Lima, Peru. Geriatricians conducted all clinical evaluations and laboratory tests were conducted in the local hospital. Participants were sought in the national vital registration system, and we collated cause (ICD-10) and date of death. We conducted a nested forward multivariate Cox proportional hazard model to identify all potential predictors of all-cause, communicable and non-communicable diseases mortality.

Results: At baseline, there were 501 older people (mean age 70.6 and 62.8\% were women), complete follow-up information was available from 427 people. Mean follow-up time was 46.5 months $(S D=25.3)$. In multivariate models, the Timed Up and Go test was associated with higher risk of all-cause mortality ( $\mathrm{HR}=1.05 ; 95 \% \mathrm{Cl}: 1.02-1.09)$. For cause-specific mortality, history of heart disease ( $\mathrm{HR}=2.25 ; 95 \% \mathrm{Cl}: 1.07-4.76)$ and age in years $(\mathrm{HR}=1.05 ; 95 \% \mathrm{Cl}$ : 1.01-1.09) were predictors of non-communicable diseases mortality.
\end{abstract}

Conclusions: In addition to established risk factors for mortality in older population, the Timed Up and Go test, a functional parameter, raised as a relevant predictor of all-cause mortality.

Keywords: Physical function, Timed up and go test, Mortality, Global health, Risk assessment

\section{Introduction}

Aging of the population is a phenomenon fast occurring in most countries. This has sparked interest in finding risk factors for death as a result of deviations of homeostatic equilibrium, deteriorations of health and multimorbidity, while considering the complexity and heterogeneity of older adults [1]. Among other risk factors, poor mobility is of great relevance and goes beyond

\footnotetext{
*Correspondence: pedro.ortiz@upch.pe

${ }^{4}$ Gerontology Institute, Universidad Peruana Cayetano Heredia, Av.

Honorio Delgado 430, San Martín de Porres, Lima, Peru

Full list of author information is available at the end of the article
}

the overall health status of the older adult affecting their independent and quality of life [2-5].

Mobility problems in older people can be detected with tests such as the Timed Up and Go test [6] and Gait Speed [7]. These are useful to predict falls [8] and to determine frailty older people [9]. Moreover, these tests are independently associated with a higher risk of mortality [7, 10-12], including mortality due to NonCommunicable Diseases (NCD) both in the presence of comorbidities and in the absence of known cardiovascular risk factors $[11,12]$. Whether this mortality risk holds in all older populations, particularly those in low- and 
middle-income countries in Latin America, where prevalence of NCD, distribution of cardiovascular risk factors and access to medical care are different than in highincome countries $[13,14]$, is unknown.

In contrast to high-income countries, high prevalence of mental and chronic diseases, mainly dementia, frailty, depression and disability [15-19] directly increase rates of well-known associated adverse outcomes including mortality. Also, older people in LMIC had lower rates of socioeconomic support and health insurance [20-22], leading to inequity, a low level of health services access and high out-of-pocket expenses to attend their health needs [23, 24]. Finally, guidelines to risk assessment for older people could not be followed, as in other frequent conditions as diabetes and hypertension [25, 26], mainly because they are not flexible, adaptable, sociocultural accepted and economically attainable.

Even the aforementioned differences in older people's characteristics of vulnerability between high-income versus LMIC and the well described role of Timed Up and Go test and Gait Speed in predict mortality in highincome countries [5, 27-29], scarce studies are done in LMIC and mainly related with all-cause mortality [30]. This evidence gap prevents recommending the application of mobility tests as a structural part of the geriatric evaluation [31]. Considering the Timed Up and Go test has the ability to explore the interactions in different systems like cardiopulmonary, nervous and musculoskeletal systems involved in it, we hypothesized that Timed $\mathrm{Up}$ and Go test is a good predictor not only for all-cause mortality, but also for NCD and non-NCD mortality.

To provide evidence to strengthen the recommendation of including mobility tests as part of the regular geriatric consultation in low- and middle-income countries particularly those in Latin America, we aimed to determine if mobility tests, such as Timed Up and Go test and Gait Speed, are independent predictors of mortality (allcauses, NCD, and due to infectious diseases/accidents), in a population-based cohort of community-dwelling older adults in Lima, Peru.

\section{Methods}

\section{Study design}

Originally, this was a cross-sectional study conducted in 2013 [19]. We turned this cross-sectional study into a prospective cohort by looking for the original participants in the vital registration system on 8th March 2020; from the vital registration system, we retrieved survival status (dead or alive), date of death and the underlying cause of death (ICD-10 code). These codes are found in an Additional file (see Additional file 1). We adhered to the Strengthening the Reporting of Observational Studies in Epidemiology (STROBE) guidelines [32] and the study was approved by the Institutional Ethics Committee for Humans at Cayetano Heredia University in Lima, Peru (Reference number: 207-06-20).

\section{Study setting}

Peru is a middle-income country located in South America. This study was developed in San Martin de Porres, the second most populated district in Lima, which is the capital of Peru. This district has a current population of 755,087 residents and $10.9 \%$ of them live in poverty [33]. Period of baseline recruitment was from January to May 2013 and the survival status was ascertained on 8th March 2020. Data collection was performed by four geriatricians through a face-to-face interview at baseline. They applied a structured questionnaire that registered demographics, socioeconomic information, and a comprehensive geriatric evaluation.

\section{Participants}

Adults older than 60 years who lived in the district of San Martín de Porres were included. A total of 501 participants were enrolled and evaluated with a health interview (e.g., self-reported diseases), a physical examination (e.g., weight and height), and laboratory tests (e.g., total cholesterol).

We followed a semi-probabilistic sampling of household clusters. San Martin de Porres was divided into eight sections, according to the distribution of sixteen primary healthcare centres and one hospital. In each section, blocks were given a random number. Every day the research team was assigned one block, until the sample size for each section was reached. In each block, households were randomly selected. In each household, all those aged $60+$ years were selected; if there were no older people living in the selected household, the adjacent home was visited.

Further details about the sampling methods and procedures of the cohort are available elsewhere [16, 19, 34]. On 8th March 2020, the national vital registration system was queried to ascertain the vital status of the participants, and when applicable, date and cause of death were retrieved as well.

\section{Variables \\ Predictors}

Participants self-reported the following information (self-reported diseases and habits): heart diseases, stroke, rheumatology diseases, respiratory diseases, tuberculosis, edentulous, insomnia, visual impairment, hearing impairment, falls, incontinence, polypharmacy and tobacco consumption.

For this analysis, diabetes mellitus, hypertension, and dyslipidaemia were determined both by self-reported 
information and laboratory tests. We considered these variables as self-reported if an older adult had a previous diagnosis of each disease or if they reported the use of oral hypoglycaemic drugs or insulin for diabetes mellitus, antihypertensive drugs for hypertension, and statins, fibrates or ezetimibe for dyslipidaemia. Furthermore, as part of laboratory assessment, we considered diabetes mellitus with fasting glucose $\geq 126 \mathrm{mg} / \mathrm{dL}$; hypertension with $\geq 140 \mathrm{mmHg}$ or $\geq 90 \mathrm{mmHg}$ for systolic and diastolic blood pressure, respectively; and (any) dyslipidaemia as total cholesterol $\geq 200 \mathrm{mg} / \mathrm{dL}$, or LDL-cholesterol $\geq 130 \mathrm{mg} / \mathrm{dL}$, or triglycerides $\geq 200 \mathrm{mg} / \mathrm{dL}$, or HDLcholesterol $\geq 50 \mathrm{mg} / \mathrm{dL}$ if the participant was female and HDL-cholesterol $\geq 40 \mathrm{mg} / \mathrm{dL}$ if the participant was male. The abdominal perimeter was measured in the physical examination. In the analysis, obesity was based on body mass index $\left(\mathrm{BMI} \geq 30 \mathrm{~kg} / \mathrm{m}^{2}\right)$ computed from measured weight and height.

Validated scales were used to determine geriatric syndromes: Pfeiffer test for Cognitive evaluation [35], Yesavage Geriatric Depression Scale for Depression [36], Barthel Index for the functional status [37], Gijon Socio-familial Evaluation Scale for social evaluation [38], malnutrition was evaluated with the Mini-Nutritional Assessment [39] and family APGAR test (adaptability, partnership, growth, affection and resolve) to determine familial dysfunction [40]. All of these instruments are validated, has a good reliability and are used as part of the Comprehensive Geriatric Assessment in Peru [41].

Functional parameters were collected using the Gait Speed test and a Timed Up and Go test; both measurements were taken using a calibrated stopwatch. Gait Speed was determined by the time required for the participant to walk $8 \mathrm{~m}$ out of a total distance of $10 \mathrm{~m}$ at the normal walking speed, without a warm-up period. The first and last meter of the walk were not considered. The shorter time between two measurements was recorded. We set two Gait Speed thresholds (1 and $1.2 \mathrm{~m} / \mathrm{second}$ ) based on prospective studies of cardiovascular events [42].

The Timed Up and Go test was assessed with the older adult sat in a chair, asked to get up, walk $3 \mathrm{~m}$, turn and return to the chair. It initiates by a cue from the measurer to get up from the chair and the measurer assessed the time it takes to sit down again. We set two Timed Up and Go test thresholds (10 and $15 \mathrm{~s}$ ) according to previously published evidence $[29,43]$.

\section{Outcomes}

The outcome was the survival status of the participants until 8th March 2020. We also studied cause-specific mortality in two groups: non-communicable diseases (NCDs) as well as accidents and infectious diseases. The
ICD-10 codes in each group are shown in an Additional file (see Additional file 1).

\section{Study sample size}

At baseline, we recruited a total of 501 participants in previous studies $[16,19,34]$. Based on that information, we calculated post-hoc sample size of 314 participants (with a power of $95 \%$ ).

\section{Statistical analysis}

Analyses were conducted with STATA SE 16.1 (StataCorp, College Station, TX, US). The statistical analysis code is available upon reasonable request.

First, characteristics of the study population were summarized using means and standard deviations (SD) or median and interquartile range (IQR) for numeric variables, depending on their distribution. Skewness and Kurtosis tests and histogram were used to assess the distribution. We did not categorize quantitative variables. To compare differences between outcome groups (alive vs death or NCDs vs accidents and infectious diseases) we used the Chi-squared or Fisher's exact tests for categorical variables; and T-test or Mann-Whitney U tests for numerical variables. Second, survival rates were analysed using the Kaplan-Meier method and the differences between groups were analysed by the log-rank test. Third, univariate analysis was performed to identify prognostic variables related to overall survival. We adjusted the Model 1 with age, sex and educational level. A fully adjusted model (Model 2) was developed with a nested forward multivariate Cox proportional hazard regression approach, considering only univariate variables with $p$ values $<0.05$. Complete-case analysis was performed throughout this work.

\section{Results}

\section{Study population}

As showed in Fig. 1, at baseline, there were 501 people, and follow-up information was available from 480 individuals; finally, 427 people with complete information in all variables of interest were herein analysed ( $89 \%$ of the study population with follow-up data).

There were more women (62.8\%), and the overall mean age was 70.6 (standard deviation: 8.5) years at baseline; most of the study population had either primary (39.6\%) or secondary (35.8\%) education, while $19.2 \%$ had higher education at baseline. Overall, the mean Gait Speed was 1.00 (standard deviation: 0.3) meters/second; similarly, the median Timed Up and Go test was 10 (interquartile range: $9-13$ ) seconds (Table 1). 


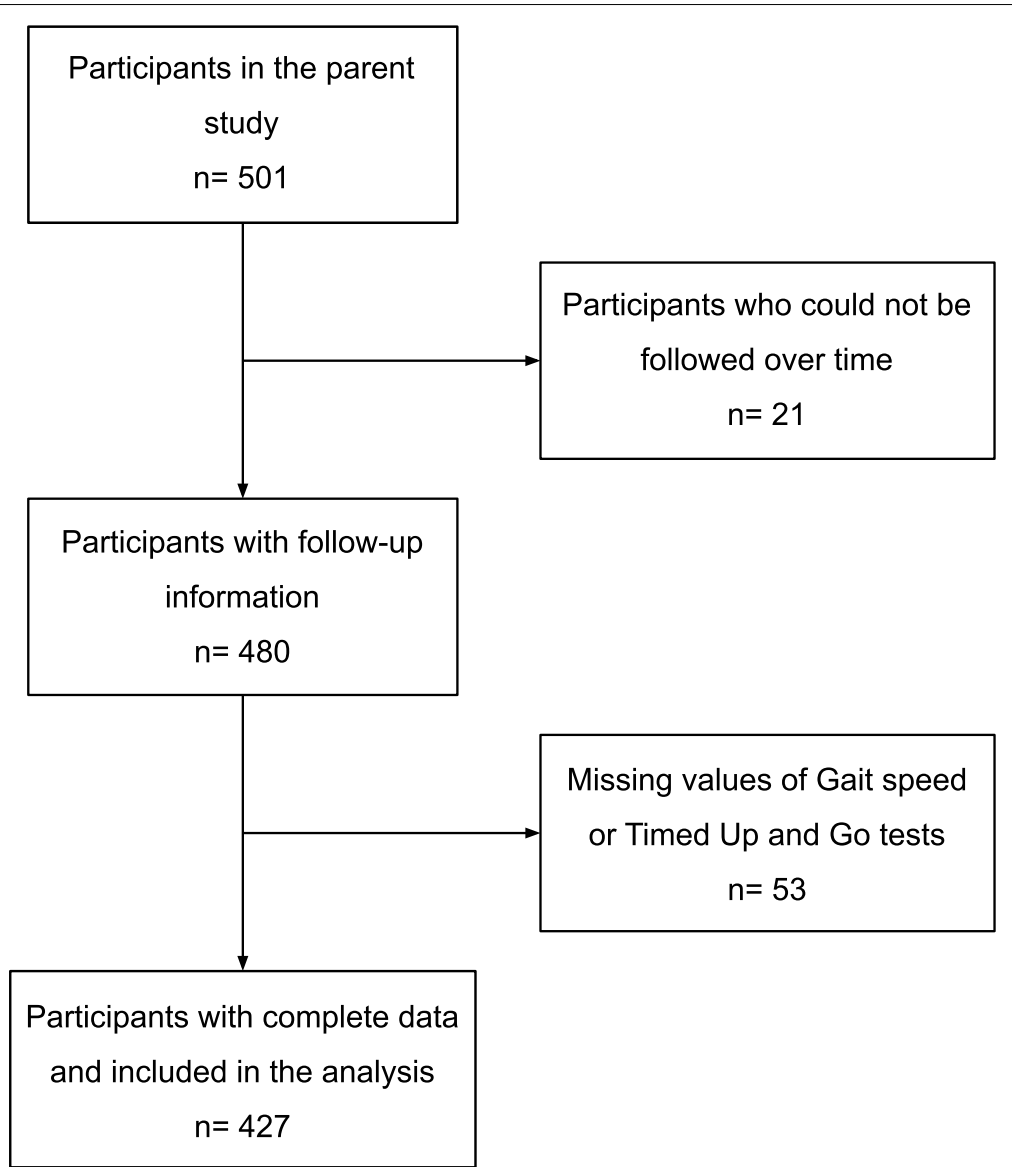

Fig. 1 Flowchart of the population included in the analysis

\section{All-cause mortality}

For all-cause mortality, the mean follow-up was 46.5 months (standard deviation: 25.3). The median Timed Up and Go test were longer among those who died (12 vs $10 \mathrm{~s} ; p<0.001)$ versus to those who survived (Table 1). We further stratified the Timed Up and Go test with thresholds at 15 and $10 \mathrm{~s}$ (Fig. $2 \mathrm{~A}$ and $\mathrm{B}$, respectively). In both cases, the survival decreased faster among those with longer Timed Up and Go test, and much faster when the Timed Up and Go test was set at $>15 \mathrm{~s}$ (Fig. 2A). Cumulative survival rates in Fig. 2 are unadjusted. The fully adjusted model revealed that the Timed Up and Go test increased the risk of all-cause mortality by 0.05 per one-unit change in seconds $(1.05,95 \% \mathrm{CI}$ : 1.02-1.09; Table 2).

The mean Gait Speed was slower in older people who died $(1.03$ vs $0.87 \mathrm{~m} /$ seconds; $p<0.001)$ versus those who survived (Table 1). We further stratified the Gait Speed with thresholds at 1.0 and $1.2 \mathrm{~m} /$ second (Fig. $2 \mathrm{C}$ and $\mathrm{D}$, respectively). With the first cut-off point $(1.0 \mathrm{~m} /$ second), the survival rate decreased faster among those who died (Fig. 2C); conversely, the survival rate did not decrease faster when the cut-off point was set at $1.2 \mathrm{~m} /$ second (Fig. 2D). The Gait Speed was not included in the adjusted Cox models; the crude analysis suggested that faster Gait Speed would be associated with lower all-cause mortality risk $(\mathrm{HR}=0.15,95 \% \mathrm{CI}$ : $0.06-0.37$; Table 2).

\section{Cause-specific mortality}

For NCDs mortality, the mean follow-up was 45.8 months (standard deviation: 26.4); for mortality due to accidents and infectious diseases the mean follow-up was 47.1 months (standard deviation: 24.7). The Timed Up and Go test was slower in older people who died of an infectious disease or an accident than in those who died of a NCD (13.0 vs $10.9 \mathrm{~s}$; Table 1$)$. In the fully adjusted model, male sex and age (in years) were associated with higher risk of dying from an infectious disease or accident (Table 3). There were several risk factors for dying of a NCD, including self-reported history of heart diseases $(\mathrm{HR}=2.25,95 \% \mathrm{CI}: 1.07-4.76$; Table 4$)$, and age in years $(\mathrm{HR}=1.05$, 95\% CI: 1.01-1.09; Table 4). 
Table 1 General characteristics of the study population

\begin{tabular}{|c|c|c|c|c|c|c|}
\hline Characteristic & $\begin{array}{l}\text { Survived } \\
N=346\end{array}$ & $\begin{array}{l}\text { Dead (all-cause) } \\
N=81\end{array}$ & $p$-value & $\begin{array}{l}\text { Dead by } \\
\text { Infections or } \\
\text { Accidents } \\
N=43\end{array}$ & $\begin{array}{l}\text { Dead by NCD } \\
N=38\end{array}$ & $p$-value \\
\hline \multicolumn{7}{|l|}{ Gender } \\
\hline Male & $117(33.82 \%)$ & $42(51.85 \%)$ & $0.003^{b}$ & $22(51.16 \%)$ & $20(52.63 \%)$ & $0.895^{b}$ \\
\hline Female & $229(66.18 \%)$ & $39(48.15 \%)$ & & $21(48.84 \%)$ & $18(47.37 \%)$ & \\
\hline Age (years); Median (IQR) & $67(63-74)$ & $76(68-83)$ & $<0.001^{\mathrm{e}}$ & $78(69-83)$ & $75.5(67-84)$ & $0.336^{e}$ \\
\hline \multicolumn{7}{|l|}{ Civil Status } \\
\hline Married or Cohabitation & $219(63.29 \%)$ & $53(65.43 \%)$ & $0.719^{b}$ & $27(62.79 \%)$ & $26(68.42 \%)$ & $0.595^{b}$ \\
\hline Single, Divorced or Widower & $127(36.71 \%)$ & $28(34.57 \%)$ & & $16(37.21 \%)$ & $12(31.58 \%)$ & \\
\hline \multicolumn{7}{|l|}{ Education } \\
\hline Higher & $68(19.65 \%)$ & $14(17.28 \%)$ & $0.200^{\mathrm{b}}$ & $6(13.95 \%)$ & $8(21.05 \%)$ & $0.235^{c}$ \\
\hline Secondary & $130(37.57 \%)$ & $23(28.40 \%)$ & & $9(20.93 \%)$ & $14(36.84 \%)$ & \\
\hline Primary & $132(38.15 \%)$ & $37(45.68 \%)$ & & $23(53.49 \%)$ & $14(36.84 \%)$ & \\
\hline No Education & $16(4.62 \%)$ & $7(8.64 \%)$ & & $5(11.63 \%)$ & $2(5.26 \%)$ & \\
\hline \multicolumn{7}{|l|}{ Functional Parameters } \\
\hline Gait Speed (m/s); Mean \pm SD & $1.03 \pm 0.25$ & $0.87 \pm 0.34$ & $<0.001^{d}$ & $0.81 \pm 0.32$ & $0.94 \pm 0.35$ & $0.081^{d}$ \\
\hline Timed Up and Go test (sec); Median (IQR) & $10(9-12)$ & $12(10-16)$ & $<0.001^{\mathrm{e}}$ & $13(10-18)$ & $10.85(9-15)$ & $0.037^{e}$ \\
\hline \multicolumn{7}{|l|}{ Clinical Variables } \\
\hline Abdominal perimeter (centimeters); Median (IQR) & $98.75(91-105)$ & $100(94-107)$ & $0.169^{e}$ & $98(91-106)$ & $101(97-108)$ & $0.191^{\mathrm{e}}$ \\
\hline Weight (kilograms); Median (IQR) & $65(56-74)$ & $65(55.5-73)$ & $0.741^{\mathrm{e}}$ & $62.5(55-70)$ & $66.8(57-75.5)$ & $0.168^{\mathrm{e}}$ \\
\hline Malnutrition & $94(27.17 \%)$ & $18(22.22 \%)$ & $0.362^{b}$ & $10(23.26 \%)$ & $8(21.05 \%)$ & $0.812^{b}$ \\
\hline Obesity & $245(70.81 \%)$ & $56(69.14 \%)$ & $0.766^{\mathrm{b}}$ & $25(58.14 \%)$ & $31(81.58 \%)$ & $0.023^{b}$ \\
\hline Dyslipidemia & $214(61.85 \%)$ & $46(56.79 \%)$ & $0.401^{b}$ & $24(55.81 \%)$ & $22(57.89 \%)$ & $0.850^{\mathrm{b}}$ \\
\hline Diabetes Mellitus ${ }^{\mathrm{a}}$ & $62(17.92 \%)$ & $13(16.05 \%)$ & $0.691^{b}$ & $7(16.28 \%)$ & $6(15.79 \%)$ & $0.952^{\mathrm{b}}$ \\
\hline Arterial Hypertension ${ }^{a}$ & $169(48.84 \%)$ & $51(62.96 \%)$ & $0.022^{b}$ & $25(58.14 \%)$ & $26(68.42 \%)$ & $0.339^{b}$ \\
\hline Heart Diseases ${ }^{\mathrm{a}}$ & $25(7.23 \%)$ & $13(16.05 \%)$ & $0.012^{b}$ & $5(11.63 \%)$ & $8(21.05 \%)$ & $0.249^{b}$ \\
\hline Stroke $e^{a}$ & $12(3.47 \%)$ & $1(1.23 \%)$ & $0.477^{c}$ & $0(0.00 \%)$ & $1(2.63 \%)$ & $0.284^{c}$ \\
\hline Rheumatological Diseases ${ }^{a}$ & $128(36.99 \%)$ & $21(25.93 \%)$ & $0.060^{b}$ & $11(25.58 \%)$ & $10(26.32 \%)$ & $0.940^{\mathrm{b}}$ \\
\hline Respiratory Diseases ${ }^{\mathrm{a}}$ & $18(5.20 \%)$ & $8(9.88 \%)$ & $0.123^{c}$ & $5(11.63 \%)$ & $3(7.89 \%)$ & $0.574^{c}$ \\
\hline Tuberculosis $^{\mathrm{a}}$ & $5(1.45 \%)$ & $3(3.70 \%)$ & $0.179^{c}$ & $2(4.65 \%)$ & $1(2.63 \%)$ & $1.000^{c}$ \\
\hline Familial dysfunction ${ }^{a}$ & $74(21.39 \%)$ & $21(25.93 \%)$ & $0.377^{b}$ & $16(37.21 \%)$ & $5(13.16 \%)$ & $0.014^{b}$ \\
\hline Sociofamily impairment & $204(58.96 \%)$ & $58(71.60 \%)$ & $0.035^{b}$ & $34(79.07 \%)$ & $24(63.16 \%)$ & $0.113^{b}$ \\
\hline Cognitive impairment & $35(10.12 \%)$ & $18(22.22 \%)$ & $0.003^{b}$ & $7(16.28 \%)$ & $11(28.95 \%)$ & $0.171^{b}$ \\
\hline Insomnia & $249(71.97 \%)$ & $63(77.78 \%)$ & $0.288^{b}$ & $33(76.74 \%)$ & $30(78.95 \%)$ & $0.812^{b}$ \\
\hline Depression & $66(19.08 \%)$ & $15(18.52 \%)$ & $0.908^{b}$ & $9(20.93 \%)$ & $6(15.79 \%)$ & $0.552^{b}$ \\
\hline Falls & $113(32.66 \%)$ & $31(38.27 \%)$ & $0.336^{b}$ & $19(44.19 \%)$ & $12(31.58 \%)$ & $0.244^{b}$ \\
\hline Edentulous & 294 (84.97\%) & $76(93.83 \%)$ & $0.035^{b}$ & $41(95.35 \%)$ & $35(92.11 \%)$ & $0.661^{c}$ \\
\hline Incontinence ${ }^{a}$ & $80(23.12 \%)$ & $19(23.46 \%)$ & $0.949^{b}$ & $11(25.58 \%)$ & $8(21.05 \%)$ & $0.631^{b}$ \\
\hline Visual problem & $259(74.86 \%)$ & $62(76.54 \%)$ & $0.752^{b}$ & $31(72.09 \%)$ & $31(81.58 \%)$ & $0.315^{b}$ \\
\hline Hearing problem & $139(40.17 \%)$ & $41(50.62 \%)$ & $0.087^{b}$ & $20(46.51 \%)$ & $21(55.26 \%)$ & $0.432^{b}$ \\
\hline Polypharmacy & $135(39.02 \%)$ & $39(48.15 \%)$ & $0.132^{b}$ & $24(55.81 \%)$ & $15(39.47 \%)$ & $0.142^{b}$ \\
\hline Tobacco Consumption & $32(9.25 \%)$ & $6(7.41 \%)$ & $0.828^{b}$ & $1(2.33 \%)$ & $5(13.16 \%)$ & $0.094^{c}$ \\
\hline
\end{tabular}

$N C D$ non-communicable disease), IQR Interquartile range, $S D$ standard deviation

a Diseases included in the table refer to self-reported history

${ }^{b}$ calculated with Chi-squared test

c calculated whit Fisher's exact test

d calculated with T-test

e calculated with Mann-Whitney $\mathrm{U}$ test 
A. Overall population per Timed Up and Go Test $>15 \mathrm{sec}$

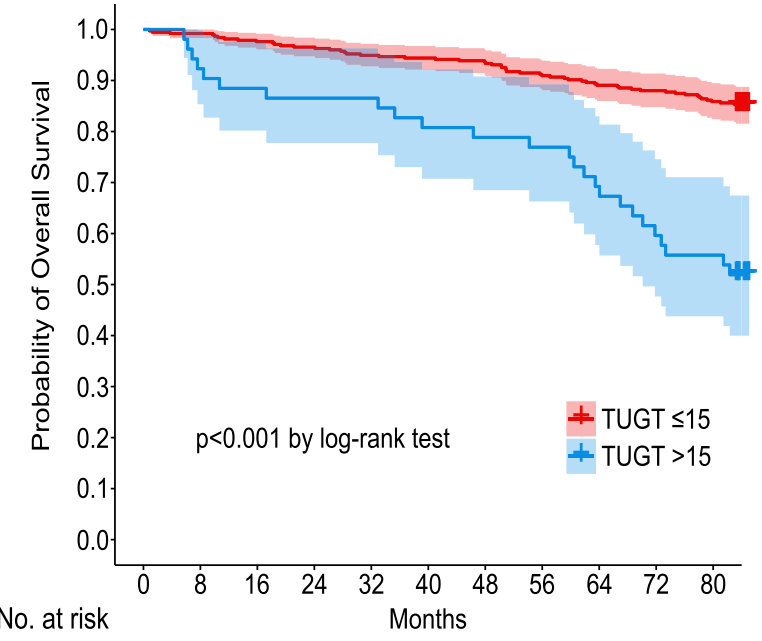

TUGT $\leq 15 \quad 375 \quad 372 \quad 366 \quad 362 \quad 356 \quad 354 \quad 351 \quad 341 \quad 334 \quad 330 \quad 322$

$\begin{array}{llllllllllll}\text { TUGT >15 } & 52 & 48 & 46 & 45 & 45 & 42 & 41 & 40 & 36 & 31 & 29\end{array}$

C. Overall population per Gait Speed $\geq 1 \mathrm{~m} / \mathrm{s}$

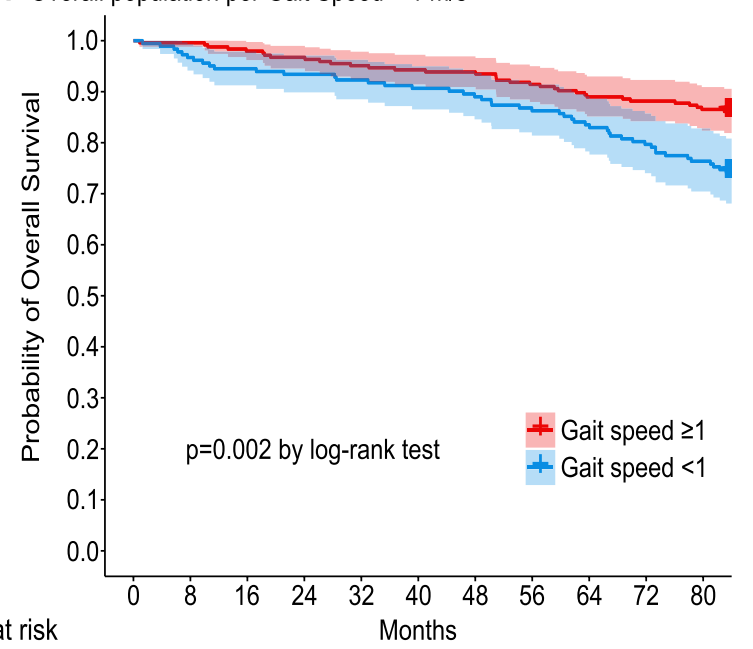

B. Overall population per Timed Up and Go Test $>10 \mathrm{sec}$

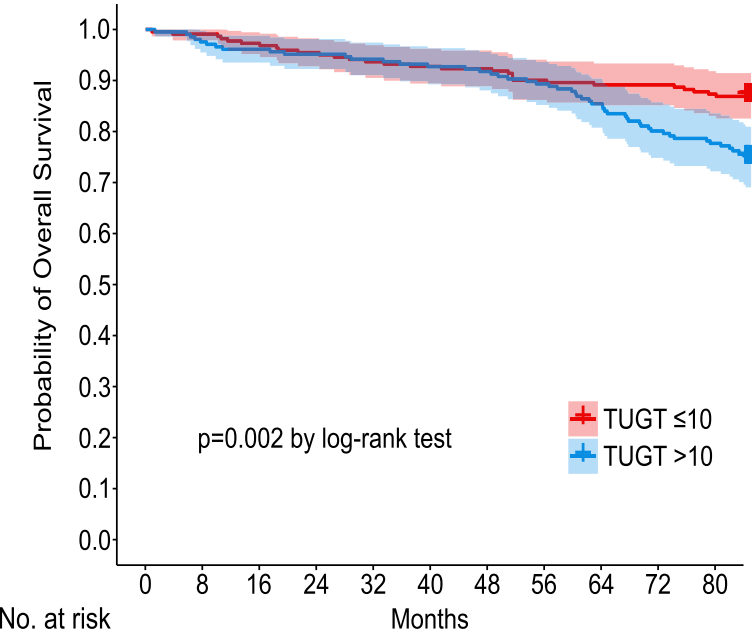

TUGT $\leq 10 \quad 221219214211207205204198 \quad 197 \quad 197 \quad 192$

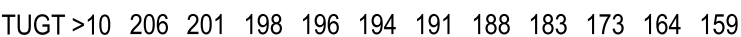

D. Overall population per Gait Speed $\geq 1.2 \mathrm{~m} / \mathrm{s}$

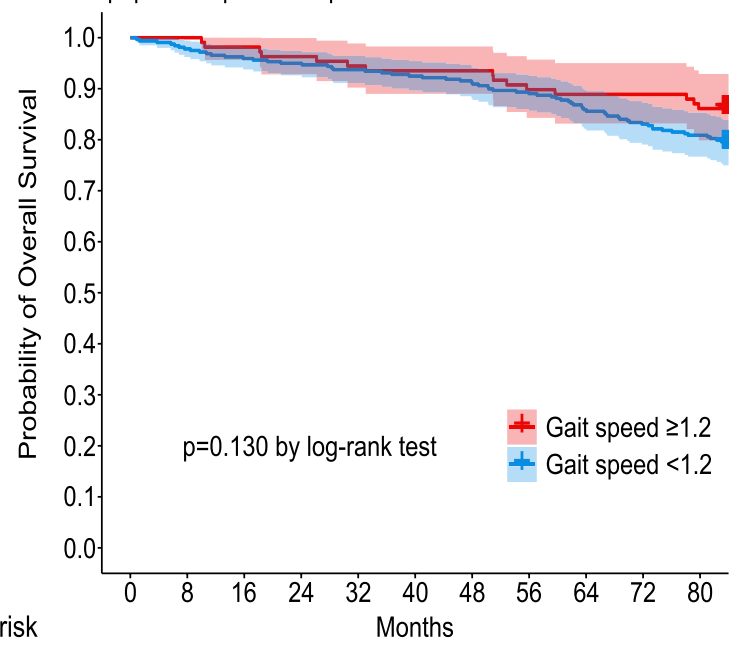

Months

Gait speed $\geq 1 \quad 245 \quad 244 \quad 240 \quad 237 \quad 233 \quad 231230224 \quad 218 \quad 216 \quad 212$

No. at risk

Gait speed $\geq 1.2 \quad 108 \quad 108 \quad 106 \quad 104 \quad 102 \quad 101 \quad 101 \quad 97 \quad 96 \quad 96 \quad 93$

Gait speed <1 $182176172 \quad 170168 \quad 165162 \quad 157 \quad 152 \quad 145 \quad 139 \quad$ Gait speed <1.2 319312306303299295291284274265258

Fig. 2 Survival curves (Kaplan-Meier) of cumulative unadjusted survival rates for the Timed Up and Go test and Gait Speed. Timed Up and Go test with a threshold of $15 \mathrm{~s}(\mathbf{A})$, for the Timed Up and Go test with a threshold of $10 \mathrm{~s}(\mathbf{B})$, for Gait Speed with a threshold of $1.0 \mathrm{~m} / \mathrm{second}(\mathbf{C})$, and for the Gait Speed with a threshold of $1.2 \mathrm{~m} / \mathrm{second}$ (D)

\section{Discussion}

\section{Main results}

In this population-based prospective cohort study of older people, and in the multivariate analysis, the Timed Up and Go test was a strong predictor of all-cause mortality, above and beyond other well-known risk factors like chronic diseases (e.g., diabetes). This suggests that the Timed Up and Go test could be part of all geriatric evaluations, in addition to the regular care and clinical assessment. Similarly, epidemiological studies could include this test in large population-based samples, to further understand its distribution and role to predict mortality. There were more risk factors strongly associated with NCDs mortality in comparison to mortality due to accidents and infectious diseases. This pinpoints the role of NCDs in the current epidemiological profile of older people living in resource-limited settings, growing apart form the idea that communicable diseases and 
Table 2 Risk factors for all-cause mortality $(N=427)$

\begin{tabular}{|c|c|c|c|c|c|c|}
\hline Characteristic & Crude Model cHR $(95 \% \mathrm{Cl})$ & $p$-value & Model 1 aHR $(95 \% \mathrm{Cl})$ & $p$-value & Model 2 aHR $(95 \% \mathrm{Cl})$ & $p$-value \\
\hline \multicolumn{7}{|l|}{ Gender } \\
\hline Male & 1 & & 1 & & 1 & \\
\hline Female & $0.51(0.33-0.78)$ & 0.002 & $0.50(0.32-0.79)$ & 0.003 & $0.52(0.33-0.80)$ & 0.003 \\
\hline Age (years) & $1.08(1.06-1.10)$ & $<0.001$ & $1.06(1.03-1.08)$ & $<0.001$ & $1.06(1.04-1.08)$ & $<0.001$ \\
\hline \multicolumn{7}{|l|}{ Civil Status } \\
\hline Married or Cohabitation & 1 & & & & & \\
\hline Single, Divorced or Widower & $0.92(0.58-1.46)$ & 0.728 & & & & \\
\hline \multicolumn{7}{|l|}{ Education } \\
\hline Higher & 1 & & 1 & & & \\
\hline Secondary & $0.84(0.43-1.64)$ & 0.605 & $0.68(0.34-1.37)$ & 0.281 & & \\
\hline Primary & $1.28(0.68-2.40)$ & 0.439 & $0.88(0.46-1.66)$ & 0.687 & & \\
\hline No Education & $1.83(0.74-4.49)$ & 0.188 & $1.04(0.34-3.11)$ & 0.950 & & \\
\hline \multicolumn{7}{|l|}{ Functional Parameters } \\
\hline Gait Speed (m/s) & $0.15(0.06-0.37)$ & $<0.001$ & & & & \\
\hline Timed Up and Go test (sec) & $1.06(1.03-1.11)$ & $<0.001$ & $1.06(1.02-1.09)$ & 0.001 & $1.05(1.02-1.09)$ & 0.001 \\
\hline \multicolumn{7}{|l|}{ Clinical Variables } \\
\hline Abdominal perimeter & $1.00(0.99-1.02)$ & 0.434 & & & & \\
\hline Weight & $0.99(0.98-1.01)$ & 0.736 & & & & \\
\hline Malnutrition & $0.80(0.47-1.35)$ & 0.399 & & & & \\
\hline Obesity & $0.91(0.57-1.47)$ & 0.704 & & & & \\
\hline Dyslipidemia ${ }^{a}$ & $0.82(0.53-1.28)$ & 0.384 & & & & \\
\hline Diabetes Mellitus ${ }^{a}$ & $0.87(0.48-1.55)$ & 0.628 & & & & \\
\hline Arterial Hypertension ${ }^{\mathrm{a}}$ & $1.65(1.05-2.60)$ & 0.030 & & & $1.43(0.91-2.25)$ & 0.117 \\
\hline Heart Diseases $^{\mathrm{a}}$ & $2.16(1.20-3.90)$ & 0.010 & & & & \\
\hline Stroke ${ }^{a}$ & $0.38(0.05-2.77)$ & 0.338 & & & & \\
\hline Rheumatological Diseases $^{\mathrm{a}}$ & $0.64(0.39-1.05)$ & 0.079 & & & & \\
\hline Respiratory Diseases $^{\mathrm{a}}$ & $1.87(0.89-3.94)$ & 0.097 & & & & \\
\hline Tuberculosis $^{\mathrm{a}}$ & $2.27(0.72-7.16)$ & 0.161 & & & & \\
\hline Familial dysfunction $^{a}$ & $1.28(0.78-2.11)$ & 0.334 & & & & \\
\hline Sociofamily impairment & $1.62(0.99-2.64)$ & 0.053 & & & & \\
\hline Cognitive impairment & $2.12(1.28-3.51)$ & 0.004 & & & & \\
\hline Insomnia ${ }^{a}$ & $1.28(0.75-2.19)$ & 0.357 & & & & \\
\hline Depression & $0.96(0.55-1.69)$ & 0.897 & & & & \\
\hline Falls & $1.24(0.80-1.95)$ & 0.338 & & & & \\
\hline Edentulous & $2.47(0.99-6.17)$ & 0.053 & & & & \\
\hline Incontinence ${ }^{a}$ & $1.04(0.62-1.75)$ & 0.878 & & & & \\
\hline Visual problem & $1.05(0.62-1.77)$ & 0.859 & & & & \\
\hline Hearing problem & $1.43(0.93-2.21)$ & 0.107 & & & & \\
\hline Polypharmacy & $1.40(0.90-2.16)$ & 0.133 & & & & \\
\hline Tobacco Consumption & $0.78(0.35-1.75)$ & $<0.548$ & & & & \\
\hline
\end{tabular}

Model 1 adjusted variables: age, sex, educational level and Timed Up and Go Test. Model 2 was developed with a nested forward multivariate Cox proportional hazard regression. cHR (crude hazard ratio), aHR (adjusted hazard ratio), 95\% Cl (95\% confidence interval)

${ }^{a}$ Diseases included in the table refer to self-reported history. Estimates in bold are statistically significant at $p<0.05$

accidents (e.g., falls) would be more relevant in these contexts.

Previous research has shown that mobility, equilibrium [44] and Timed Up and Go test scores are affected by advanced age [45]. The rise in the prevalence of medical and health conditions associated with the aging process will affect functional tests [46] and the Timed Up and Go test has the ability to reflect the 
Table 3 Risk factors for mortality of accidents and infectious diseases ( $N=389)$

\begin{tabular}{|c|c|c|c|c|c|c|}
\hline Characteristic & Crude Model cHR $(95 \% \mathrm{Cl})$ & $p$-value & Model 1 aHR $(95 \% \mathrm{Cl})$ & $p$-value & Model 2 aHR $(95 \% \mathrm{Cl})$ & $p$-value \\
\hline \multicolumn{7}{|l|}{ Gender } \\
\hline Male & 1 & & 1 & & 1 & \\
\hline Female & $0.50(0.28-0.91)$ & 0.024 & $0.41(0.22-0.79)$ & 0.007 & $0.37(0.20-0.70)$ & 0.002 \\
\hline Age (years) & $1.10(1.07-1.13)$ & $<0.001$ & $1.06(1.02-1.10)$ & 0.002 & $1.06(1.02-1.10)$ & 0.001 \\
\hline \multicolumn{7}{|l|}{ Civil Status } \\
\hline Married or Cohabitation & 1 & & & & & \\
\hline Single, Divorced or Widower & $1.02(0.55-1.90)$ & 0.939 & & & & \\
\hline \multicolumn{7}{|l|}{ Education } \\
\hline Higher & 1 & & 1 & & & \\
\hline Secondary & $0.78(0.28-2.20)$ & 0.643 & $0.55(0.18-1.68)$ & 0.294 & & \\
\hline Primary & $1.89(0.76-4.65)$ & 0.169 & $1.24(0.49-3.14)$ & 0.651 & & \\
\hline No Education & $3.20(0.97-10.52)$ & 0.055 & $1.55(0.35-6.74)$ & 0.562 & & \\
\hline \multicolumn{7}{|l|}{ Functional Parameters } \\
\hline Gait Speed (m/s) & $0.06(0.02-0.19)$ & $<0.001$ & & & $0.28(0.06-1.25)$ & 0.097 \\
\hline Timed Up and Go test (sec) & $1.08(1.04-1.13)$ & $<0.001$ & $1.08(1.04-1.13)$ & $<0.001$ & $1.04(0.99-1.09)$ & 0.158 \\
\hline \multicolumn{7}{|l|}{ Clinical Variables } \\
\hline Abdominal perimeter & $1.00(0.98-1.02)$ & 0.833 & & & & \\
\hline Weight & $0.98(0.96-1.01)$ & 0.172 & & & & \\
\hline Malnutrition & $0.83(0.41-1.70)$ & 0.614 & & & & \\
\hline Obesity & $0.58(0.32-1.06)$ & 0.079 & & & & \\
\hline Dyslipidemia $^{a}$ & $0.79(0.43-1.44)$ & 0.445 & & & & \\
\hline Diabetes Mellitus ${ }^{a}$ & $0.87(0.39-1.93)$ & 0.738 & & & & \\
\hline Arterial Hypertension ${ }^{\mathrm{a}}$ & $1.42(0.78-2.60)$ & 0.255 & & & & \\
\hline Heart Diseases $^{\mathrm{a}}$ & $1.67(0.65-4.29)$ & 0.291 & & & & \\
\hline Stroke & $0.00(0.00-0.00)^{b}$ & $<0.001$ & & & & \\
\hline Rheumatological Diseases $^{\mathrm{a}}$ & $0.61(0.31-1.21)$ & 0.159 & & & & \\
\hline Respiratory Diseases ${ }^{a}$ & $2.21(0.88-5.56)$ & 0.092 & & & & \\
\hline Tuberculosis $^{\mathrm{a}}$ & $2.89(0.72-11.56)$ & 0.134 & & & & \\
\hline Familial dysfunction ${ }^{\mathrm{a}}$ & $2.11(1.13-3.92)$ & 0.019 & & & & \\
\hline Sociofamily impairment & $2.47(1.18-5.18)$ & 0.017 & & & $1.99(0.91-4.36)$ & 0.087 \\
\hline Cognitive impairment & $1.67(0.74-3.74)$ & 0.216 & & & & \\
\hline Insomnia & $1.23(0.60-2.53)$ & 0.569 & & & & \\
\hline Depression & $1.11(0.54-2.32)$ & 0.774 & & & & \\
\hline Falls & $1.58(0.87-2.89)$ & 0.134 & & & & \\
\hline Edentulous & $3.45(0.83-14.30)$ & 0.088 & & & & \\
\hline Incontinence ${ }^{a}$ & $1.16(0.58-2.30)$ & 0.682 & & & & \\
\hline Visual problem & $0.86(0.44-1.68)$ & 0.652 & & & & \\
\hline Hearing problem & $1.27(0.70-2.30)$ & 0.441 & & & & \\
\hline Polypharmacy & $1.93(1.06-3.52)$ & 0.031 & & & $1.68(0.90-3.14)$ & 0.102 \\
\hline Tobacco Consumption & $0.24(0.03-1.71)$ & 0.154 & & & & \\
\hline
\end{tabular}

Model 1 adjusted variables: age, sex, educational level and Timed Up and Go Test. Model 2 was developed with a nested forward multivariate Cox proportional hazard regression. cHR (crude hazard ratio), aHR (adjusted hazard ratio), $95 \% \mathrm{Cl}$ ( $95 \%$ confidence interval)

a , Diseases included in the table refer to self-reported history

b , Values were approximated $\left(\mathrm{cHR}=1.63 \times 10^{-15}, 95 \% \mathrm{Cl}: 8.57 \times 10^{-16}-3.09 \times 10^{-15}\right)$. Estimates in bold are statistically significant at $p<0.05$

burden of multimorbidity in different body systems that participate in coordination, mobility and balance [27]. The main mechanism suggested of the relationship of the increasing risk of mortality with an advancing age was through the development of multiple comorbidities that produce a poor physical performance [11].

Certainly, age is a variable that influences Timed Up and Go test and some reports have demonstrated that sex 
Table 4 Risk factors for mortality of non-communicable diseases $(N=384)$

\begin{tabular}{|c|c|c|c|c|c|c|}
\hline Characteristic & $\begin{array}{l}\text { Crude Model } \\
\text { cHR }(95 \% \mathrm{Cl})\end{array}$ & $p$-value & Model 1 aHR $(95 \% \mathrm{Cl})$ & $p$-value & Model 2 aHR $(95 \% \mathrm{Cl})$ & $p$-value \\
\hline \multicolumn{7}{|l|}{ Gender } \\
\hline Male & 1 & & 1 & & 1 & \\
\hline Female & $0.48(0.25-0.91)$ & 0.024 & $0.54(0.28-1.05)$ & 0.071 & $0.50(0.27-0.92)$ & 0.027 \\
\hline Age (years) & $1.07(1.04-1.11)$ & $<0.001$ & $1.07(1.03-1.11)$ & 0.001 & $1.05(1.01-1.09)$ & 0.026 \\
\hline \multicolumn{7}{|l|}{ Civil Status } \\
\hline Married or Cohabitation & 1 & & & & & \\
\hline Single, Divorced or Widower & $0.80(0.41-1.59)$ & 0.529 & & & & \\
\hline \multicolumn{7}{|l|}{ Education } \\
\hline Higher & 1 & & 1 & & & \\
\hline Secondary & $0.89(0.37-2.15)$ & 0.800 & $0.79(0.32-1.95)$ & 0.606 & & \\
\hline Primary & $0.89(0.37-2.14)$ & 0.792 & $0.63(0.27-1.49)$ & 0.292 & & \\
\hline No Education & $1.01(0.22-4.60)$ & 0.993 & $0.53(0.08-3.45)$ & 0.510 & & \\
\hline \multicolumn{7}{|l|}{ Functional Parameters } \\
\hline Gait Speed (m/s) & $0.31(0.07-1.43)$ & 0.133 & & & & \\
\hline Timed Up and Go test (sec) & $1.05(1.01-1.10)$ & 0.016 & $1.03(0.98-1.09)$ & 0.227 & $1.01(0.96-1.06)$ & 0.764 \\
\hline \multicolumn{7}{|l|}{ Clinical Variables } \\
\hline Abdominal perimeter & $1.02(0.99-1.05)$ & 0.139 & & & & \\
\hline Weight & $1.01(0.99-1.03)$ & 0.407 & & & & \\
\hline Malnutrition & $0.73(0.33-1.59)$ & 0.430 & & & & \\
\hline Obesity & $1.77(0.78-4.03)$ & 0.171 & & & & \\
\hline Dyslipidemia $^{a}$ & $0.85(0.45-1.62)$ & 0.618 & & & & \\
\hline Diabetes Mellitus ${ }^{\mathrm{a}}$ & $0.87(0.36-2.08)$ & 0.749 & & & & \\
\hline Arterial Hypertension ${ }^{a}$ & $2.14(1.08-4.26)$ & 0.030 & & & $1.68(0.85-3.33)$ & 0.137 \\
\hline Heart Diseases $^{\mathrm{a}}$ & $3.01(1.40-6.46)$ & 0.005 & & & $2.25(1.07-4.76)$ & 0.033 \\
\hline Stroke ${ }^{a}$ & $0.77(0.10-5.60)$ & 0.792 & & & & \\
\hline Rheumatological Diseases ${ }^{\mathrm{a}}$ & $0.63(0.30-1.29)$ & 0.204 & & & & \\
\hline Respiratory Diseases $^{\mathrm{a}}$ & $1.57(0.47-5.27)$ & 0.452 & & & & \\
\hline Tuberculosis $^{\mathrm{a}}$ & $1.76(0.25-12.68)$ & 0.573 & & & & \\
\hline Familial dysfunction ${ }^{\mathrm{a}}$ & $0.57(0.22-1.46)$ & 0.243 & & & & \\
\hline Sociofamily impairment & $1.17(0.60-2.26)$ & 0.646 & & & & \\
\hline Cognitive impairment & $3.09(1.56-6.11)$ & 0.001 & & & $1.89(0.90-3.96)$ & 0.093 \\
\hline Insomnia ${ }^{a}$ & $1.43(0.65-3.12)$ & 0.371 & & & & \\
\hline Depression & $0.80(0.34-1.92)$ & 0.624 & & & & \\
\hline Falls & $0.95(0.48-1.89)$ & 0.891 & & & & \\
\hline Edentulous & $1.99(0.61-6.51)$ & 0.255 & & & & \\
\hline Incontinence ${ }^{a}$ & $0.90(0.41-1.97)$ & 0.790 & & & & \\
\hline Visual problem & $1.44(0.63-3.12)$ & 0.386 & & & & \\
\hline Hearing problem & $1.76(0.93-3.33)$ & 0.084 & & & & \\
\hline Polypharmacy & $1.00(0.52-1.90)$ & 0.989 & & & & \\
\hline Tobacco Consumption & $1.43(0.56-3.60)$ & 0.452 & & & & \\
\hline
\end{tabular}

Model 1 adjusted variables: age, sex, educational level and Timed Up and Go Test. Model 2 was developed with a nested forward multivariate Cox proportional hazard regression. CHR (crude hazard ratio), aHR (adjusted hazard ratio), $95 \% \mathrm{Cl}$ (95\% confidence interval)

${ }^{a}$ Diseases included in the table refer to self-reported history. Estimates in bold are statistically significant at $p<0.05$

and BMI also affects Timed Up and Go test [5]. Nevertheless, Timed Up and Go test was an all-cause mortality predictor independent of age, which gives a window of opportunity for screening and intervention. While there is nothing we can do to stop aging, we could incorporate the Timed Up and Go test in the regular geriatric consultation, and intervene to improve the reasons for poor performance in this test. 


\section{Results in context}

The Timed Up and Go test was associated with higher risk of all-cause mortality. This goes in line with previous reports signalling that non-optimal results in the Timed Up and Go test increased the risk of all-cause mortality in the older population in high income countries by $20-60 \%$ [27, 47]; of note, these risk estimates would be higher when the underlying population had history of cardiovascular diseases or were women [29, 47]. The risk magnitude for all-cause mortality herein quantified was lower than that of these studies [27, 47], and there are potential explanations. First, these previous studies were conducted with a longer follow-up time contributing to detect more events (deaths) thus a stronger association. Second, we studied a population of Peru, a middle income country in Latin America where life expectancy increased into 2.3 years between 2005 and 2015 [48] compared with other countries $[27,47]$ where life expectancy increases just into 1.2-2.1 years in the same period [48].

The Timed Up and Go test is recommended as a routine screening test for falls [49], and its usefulness as a predictor of low physical performance and adverse events has been described $[43,47,50]$. It has been suggested that poor performance in the Timed Up and Go test is associated with higher mortality risk because it reflects underlying malaise, sarcopenia and chronic illness [27], all of which affects mobility, balance, strength and gait. The Timed Up and Go test is a more complex task that assess all these functions of mobility and strength, which could explain why it is a better mortality predictor than other features of the formal geriatric assessment [29]. Our results contribute and advance these recommendations by showing that the Timed Up and Go test is also associated with all-cause mortality. In so doing, we could suggest implementing the Timed Up and Go test as a regular screening test in older population, and not only to look for those at higher risk of falls.

Another important functional parameter in older population is the Gait Speed, and our results suggested, though with non-significant results in the adjusted models, that faster Gait Speed would reduce the risk of dying from communicable diseases and accidents. In the literature, poor Gait Speed has been associated with higher risk of all-cause mortality $[51,52]$, and it seems to be as good a tool as Timed Up and Go test to predict adverse events [43]. The reason because our results did not show a strong association could be lack of statistical power; small sample or few outcome events. In any case, our results provide preliminary evidence, pending further research, that the Gait Speed could also be incorporated as a standard screening test in older people care.

In our study, the Timed Up and Go test demonstrate to be a relevant mortality predictor, even independently of other socio-demographic traits and medical background. This functional test could be assessed more often in clinical evaluations and regular check-ups among older population in resource-limited countries. Future studies should assess the net benefit of including this test as standard and frequent care of the older population. Finally, talking about cause-specific mortality, history of heart diseases demonstrated to increase the risk for mortality of NCDs. Evidence showed that history of heart diseases increases the risk of cardiovascular disease associated with metabolic syndrome [53] and could contribute in this way to higher mortality risk due to NCDs.

\section{Strengths and limitations}

This is a population-based prospective cohort study of people aged $\geq 60$ in a resource-limited environment in Lima, Peru. Prospective research in gerontology, and in general addressing the wellbeing of older populations lack, particularly in low- and middle-income countries [31]. Our work contributed to this research field signalling the strong association with mortality of the Timed Up and Go test, above and beyond other stablished risk factors. All predictors at baseline were collected by trained geriatricians, and blood tests were analysed in one laboratory. Mortality information was based on death certificates, and not on reported information by a family member without further verification.

Nonetheless, there are limitations we acknowledge. First, although some information was collected by trained physicians with a standard questionnaire following a strict protocol, this information remains selfreported and could be biased (e.g., recall bias); this information would also depend on whether the participant is aware they have the condition or not. As we did for diabetes, hypertension and dyslipidaemia which were based on both self-reported and objective assessments, future work should verify our results with a more robust ascertainment of all self-reported predictors. Second, the number of outcome events was still limited to further inspect specific mortality causes (e.g., ischaemic heart disease versus stroke). Larger cohorts could provide this evidence, and future follow-ups of our cohort will also give lights about this. Additionally, hazard ratio values of stroke in Table 3 were too small, approximately zero. We believe this was a result of no stroke events in older adults who died from an infectious disease or accident; this estimate should not be interpreted as a significant finding. Third, confounding bias could influence the association between the functional parameters (Gait speed and Timed Up and Go tests) and mortality. Trying to minimize this bias, we considered multiple covariates as comorbidities, cognitive impairment, polypharmacy 
and visual problems in the analysis; however, residual confounding could not be ruled out due to inherent data. Fourth, we studied mortality, which is an extreme outcome. Unfortunately, it was not possible to use registry data to study other non-fatal (e.g., non-fatal myocardial infarction) and intermediate (e.g., emergency visits) outcomes; similarly, it was not possible to study healthy aging or functional decline. Future work, with an active face-to-face follow-up of the original participants could provide this information.

\section{Conclusions}

The Timed Up and Go test was a strong all-cause mortality predictor, displacing other stablished risk factors like chronic diseases. This could support the recommendation to consistently include the Timed Up and Go test in all geriatric consultations. Likewise, this could suggest the introduction of this test in national and epidemiological large-scale surveys studying the wellbeing of the older people.

\section{Abbreviations}

NCD: Non-Communicable Diseases; ICD-10: International Classification of Diseases; BMI: Body mass index; YLD: Years lived with disability; DALYs: Disabilityadjusted life-year.

\section{Supplementary Information}

The online version contains supplementary material available at https://doi. org/10.1186/s12877-022-02749-6.

Additional file 1. ICD-10 codes for Non-communicable disease and Infectious diseases or Accident cause of death.

\section{Acknowledgements}

The authors are grateful to all the geriatricians of the Gerontology Institute of the Universidad Peruana Cayetano Heredia, who perform the geriatric assessment and collect data for this research.

\section{Authors' contributions}

All authors designed the study. GDC-G and PJO performed the data collection. EJA and RMC-L analysed the data. All authors drafted the manuscript and approved the final manuscript.

\section{Funding}

RMC-L is supported by a Wellcome Trust International Training Fellowship (214185/Z/18/Z).

\section{Availability of data and materials}

The datasets used and analysed during the current study are available from the corresponding author on reasonable request.

\section{Declarations}

\section{Ethics approval and consent to participate}

All participants in the original cohort study gave informed consent and the study was approved by the Institutional Ethics Committee for Humans at Cayetano Heredia University in Lima, Peru (Reference number: 207-06-20). All methods in the study were performed in accordance with guidelines and regulations of the Declaration of Helsinki.
Consent for publication

Not applicable.

\section{Competing interests}

The authors declare no conflict of interests.

\section{Author details}

${ }^{1}$ School of Medicine, Universidad Peruana Cayetano Heredia, Lima, Peru. ${ }^{2}$ CRONICAS Centre of Excellence in Chronic Diseases, Universidad Peruana Cayetano Heredia, Lima, Peru. ${ }^{3}$ Department of Epidemiology and Biostatistics, School of Public Health, Imperial College London, London, UK. ${ }^{4}$ Gerontology Institute, Universidad Peruana Cayetano Heredia, Av. Honorio Delgado 430, San Martín de Porres, Lima, Peru.

Received: 27 May 2021 Accepted: 20 December 2021

Published online: 18 January 2022

\section{References}

1. Ferrucci L, Kuchel GA. Heterogeneity of aging: individual risk factors, mechanisms, patient priorities, and outcomes. J Am Geriatr Soc. 2021;69(3):610-2. https://doi.org/10.1111/jgs.17011.

2. Hirvensalo M, Rantanen T, Heikkinen E. Mobility difficulties and physical activity as predictors of mortality and loss of Independence in the community-living older population. J Am Geriatr Soc. 2000;48(5):493-8. https://doi.org/10.1111/j.1532-5415.2000.tb04994.x PubMed PMID: 10811541. Epub 2000/05/16.

3. Musich S, Wang SS, Ruiz J, Hawkins K, Wicker E. The impact of mobility limitations on health outcomes among older adults. Geriatric Nurs (New York, NY). 2018;39(2):162-9. https://doi.org/10.1016/j.gerinurse.2017.08. 002 PubMed PMID: 28866316. Epub 2017/09/04.

4. Olaya B, Moneta MV, Doménech-Abella J, Miret M, Bayes I, Ayuso-Mateos $J \mathrm{~L}$, et al. Mobility difficulties, physical activity, and all-cause mortality risk in a nationally representative sample of older adults. J Gerontol A Biol Sci Med Sci. 2018;73(9):1272-9. https://doi.org/10.1093/gerona/glx121 PubMed PMID: 28633439. Epub 2017/06/22.

5. Otsuka H, Kobayashi H, Suzuki K, Hayashi Y, Ikeda J, Kushimoto M, et al. Mobility performance impacts mortality risk in community-dwelling healthy older adults in Japan: a prospective observational study. Aging Clin Exp Res. 2021;33(9):2511-7. https://doi.org/10.1007/s40520-02101787-1 PubMed PMID: 33496935. Epub 2021/01/27.

6. Podsiadlo D, Richardson S. The timed "up \& go": a test of basic functional mobility for frail elderly persons. J Am Geriatr Soc. 1991;39(2):142-8. https://doi.org/10.1111/j.1532-5415.1991.tb01616.x PubMed PMID: 1991946. Epub 1991/02/01

7. Studenski S. Gait speed and survival in older adults. Jama. 2011;305(1):508. https://doi.org/10.1001/jama.2010.1923.

8. Shumway-Cook A, Brauer S, Woollacott M. Predicting the probability for falls in community-dwelling older adults using the timed up \& go test. Phys Ther. 2000;80(9):896-903 Epub 2000/08/29. PubMed PMID: 10960937.

9. Fried LP, Tangen CM, Walston J, Newman AB, Hirsch C, Gottdiener J, et al. Frailty in older adults: evidence for a phenotype. J Gerontol Ser A Biol Med Sci. 2001;56(3):M146-M57. https://doi.org/10.1093/gerona/56.3. M146 PubMed PMID: 11253156. Epub 2001/03/17.

10. Cesari M, Kritchevsky SB, Penninx BWHJ, Nicklas BJ, Simonsick EM, Newman $A B$, et al. Prognostic value of usual gait speed in well-functioning older People-results from the health, aging and body composition study. J Am Geriatr Soc. 2005;53(10):1675-80. https://doi.org/10.1111/j.15325415.2005.53501.x PubMed PMID: 16181165. Epub 2005/09/27.

11. Chun S, Shin DW, Han K, Jung JH, Kim B, Jung H-W, et al. The timed up and go test and the ageing heart: findings from a national health screening of 1,084,875 community-dwelling older adults. Eur J Prev Cardiol. 2021;28(2):213-9. https://doi.org/10.1177/2047487319882118 PubMed PMID: 33838038. Epub 2021/04/11.

12. Vetrano DL, Rizzuto D, Calderón-Larrañaga A, Onder G, Welmer A-K, Qiu C, et al. Walking Speed Drives the Prognosis of Older Adults with Cardiovascular and Neuropsychiatric Multimorbidity. Am J Med. 2019;132(10):1207-15.e6. Epub 2019/05/31. PubMed PMID: 31145879. https://doi.org/10.1016/j.amjmed.2019.05.005. 
13. Federici S, Flores-Flores O, Bell R, Reynolds R, Bernabé-Ortiz A. Older adults with disability in extreme poverty in Peru: how is their access to health care? PLoS One. 2018;13(12):e0208441. https://doi.org/10.1371/ journal.pone.0208441.

14. Lozano R, Naghavi M, Foreman K, Lim S, Shibuya K, Aboyans V, et al. Global and regional mortality from 235 causes of death for 20 age groups in 1990 and 2010: a systematic analysis for the global burden of disease study 2010. Lancet. 2012;380(9859):2095-128. https://doi.org/10.1016/ s0140-6736(12)61728-0.

15. Nguyen T, Cumming RG, Hilmer SN. A review of frailty in developing countries. J Nutr Health Aging. 2015;19(9):941-6. https://doi.org/10.1007/ s12603-015-0503-2 PubMed PMID: 26482697. Epub 2015/10/21.

16. Ortiz PJ, Tello T, Aliaga EG, Casas PM, Peinado JE, Miranda JJ, et al. Effect of multimorbidity on gait speed in well-functioning older people: a population-based study in Peru. Geriatr Gerontol Int. 2018;18(2):293-300. https://doi.org/10.1111/ggi.13182 PubMed PMID: 29076226. Epub 2017/10/28.

17. Prina AM, Stubbs B, Veronese N, Guerra M, Kralj C, Llibre Rodriguez JJ, et al. Depression and Incidence of Frailty in Older People From Six Latin American Countries. Am J Geriatric Psychiatry. 2019;27(10):1072-9. https://doi.org/10.1016/j.jagp.2019.04.008 PubMed PMID: 31109899; PubMed Central PMCID: PMCPMC6742503. Epub 2019/05/22.

18. Vancampfort D, Lara E, Smith L, Rosenbaum S, Firth J, Stubbs B, et al. Physical activity and loneliness among adults aged 50 years or older in six low- and middle-income countries. Int J Geriatric Psychiatry. 2019;34(12):1855-64. https://doi.org/10.1002/gps.5202 PubMed PMID: 31435958; PubMed Central PMCID: PMCPMC6854283. Epub 2019/08/23.

19. Varela-Pinedo L, Chavez-Jimeno H, Tello-Rodriguez T, Ortiz-Saavedra P, Galvez-Cano M, Casas-Vasquez P, et al. Clinical, functional and older socio-familiar profile of the community in a district of Lima, Peru. Revista peruana de medicina experimental y salud publica. 2015;32(4):709-16 Epub 2016/01/07. PubMed PMID: 26732919.

20. Hoogendijk EO, Rijnhart JJM, Kowal P, Pérez-Zepeda MU, Cesari M, Abizanda $\mathrm{P}$, et al. Socioeconomic inequalities in frailty among older adults in six low- and middle-income countries: results from the WHO study on global AGEing and adult health (SAGE). Maturitas. 2018;115:56-63. https://doi.org/10.1016/j.maturitas.2018.06.011 PubMed PMID: 30049348. Epub 2018/07/28.

21. Prince MJ, Lloyd-Sherlock P, Guerra M, Huang Y, Sosa AL, Uwakwe R, et al. The economic status of older people's households in urban and rural settings in Peru, Mexico and China: a 10/66 INDEP study crosssectional survey. SpringerPlus. 2016;5(1):258. https://doi.org/10.1186/ s40064-016-1913-2 PubMed PMID: 27006867; PubMed Central PMCID: PMCPMC4775717. Epub 2016/03/24.

22. van Hees SGM, O'Fallon T, Hofker M, Dekker M, Polack S, Banks LM, et al. Leaving no one behind? Social inclusion of health insurance in lowand middle-income countries: a systematic review. Int J Equity Health. 2019;18(1):134. https://doi.org/10.1186/s12939-019-1040-0 PubMed PMID: 31462303; PubMed Central PMCID: PMCPMC6714392. Epub 2019/08/30.

23. Albanese E, Liu Z, Acosta D, Guerra M, Huang Y, Jacob KS, et al. Equity in the delivery of community healthcare to older people: findings from 10/66 Dementia Research Group cross-sectional surveys in Latin America, China, India and Nigeria. BMC Health Serv Res. 2011;11(1):153. https:// doi.org/10.1186/1472-6963-11-153 PubMed PMID: 21711546; PubMed Central PMCID: PMCPMC3146820. Epub 2011/06/30.

24. Hernández-Vásquez A, Rojas-Roque C, Santero M, Prado-Galbarro FJ, Rosselli D. Gasto de bolsillo en salud en adultos mayores peruanos: análisis de la Encuesta Nacional de Hogares sobre Condiciones de Vida y Pobreza 2017. Revista peruana de medicina experimental y salud publica. 2018;35(3):390-9. https://doi.org/10.17843/rpmesp.2018.353.3815 PubMed PMID: 30517498. Epub 2018/12/06.

25. Owolabi M, Olowoyo P, Miranda JJ, Akinyemi R, Feng W, Yaria J, et al. Gaps in Hypertension Guidelines in Low- and Middle-Income Versus HighIncome Countries. Hypertension (Dallas, Tex : 1979). 2016;68(6):1328-37. https://doi.org/10.1161/hypertensionaha.116.08290 PubMed PMID: 27698059; PubMed Central PMCID: PMCPMC5159303. Epub 2016/10/05.

26. Owolabi MO, Yaria JO, Daivadanam M, Makanjuola Al, Parker G, Oldenburg B, et al. Gaps in Guidelines for the Management of Diabetes in Low- and Middle-Income Versus High-Income Countries-A Systematic Review. Diabetes Care. 2018;41(5):1097-105. https://doi.org/10. 2337/dc17-1795 PubMed PMID: 29678866; PubMed Central PMCID: PMCPMC5911785. Epub 2018/04/22.

27. Bergland A, Jørgensen L, Emaus N, Strand BH. Mobility as a predictor of all-cause mortality in older men and women: 11.8 year follow-up in the Tromsø study. BMC Health Serv Res. 2017;17(1):22. https://doi.org/ 10.1186/s12913-016-1950-0 PubMed PMID: 28068995; PubMed Central PMCID: PMCPMC5223479. Epub 2017/01/11.

28. Chua KY, Lim WS, Lin X, Yuan JM, Koh W-P. Handgrip strength and timed up-and-go (TUG) test are predictors of short-term mortality among elderly in a population-based cohort in Singapore. J Nutr Health Aging. 2020;24(4):371-8. https://doi.org/10.1007/s12603-020-1337-0 PubMed PMID: 32242204. Epub 2020/04/04.

29. Son KY, Shin DW, Lee JE, Kim SH, Yun JM, Cho B. Association of timed up and go test outcomes with future incidence of cardiovascular disease and mortality in adults aged 66 years: Korean national representative longitudinal study over 5.7 years. BMC Geriatr. 2020;20(1):111. https:// doi.org/10.1186/s12877-020-01509-8 PubMed PMID: 32192437; PubMed Central PMCID: PMCPMC7081542. Epub 2020/03/21.

30. At J, Bryce R, Prina M, Acosta D, Ferri CP, Guerra M, et al. Frailty and the prediction of dependence and mortality in low- and middleincome countries: a 10/66 population-based cohort study. BMC Med. 2015;13(1):138. https://doi.org/10.1186/s12916-015-0378-4.

31. Gutierrez-Robledo LM. Looking at the future of geriatric Care in Developing Countries. J Gerontol Ser A Biol Med Sci. 2002;57(3):M162-M7. https:// doi.org/10.1093/gerona/57.3.M162 PubMed PMID: 11867652. Epub 2002/02/28.

32. Vandenbroucke JP, von Elm E, Altman DG, Gøtzsche PC, Mulrow CD, Pocock SJ, et al. Strengthening the Reporting of Observational Studies in Epidemiology (STROBE): explanation and elaboration. PLOS Med. 2007;4(10):e297. https://doi.org/10.1371/journal.pmed.0040297 PubMed PMID: 17941715; PubMed Central PMCID: PMCPMC2020496. Epub 2007/10/19.

33. Ministerio de Salud del Peru. Estadística Poblacional 2021; https://public. tableau.com/profile/ogei.minsa.peru\#!/vizhome/Poblacionestimada/ INICIO?publish=yes. Accessed 03 Apr 2021.

34. Rodríguez G, Burga-Cisneros D, Cipriano G, Ortiz PJ, Tello T, Casas P, et al. Factores asociados a velocidad de marcha lenta en adultos mayores de un distrito en Lima, Perú. Revista peruana de medicina experimental y salud publica. 2017;34(4):619-26. https://doi.org/10.17843/rpmesp.2017. 344.3025 PubMed PMID: 29364417. Epub 2018/01/25.

35. Pfeiffer E. A short portable mental status questionnaire for the assessment of organic brain deficit in elderly patientst. J Am Geriatr Soc. 1975;23(10):433-41. https://doi.org/10.1111/j.1532-5415.1975.tb00927.x PubMed PMID: 1159263. Epub 1975/10/01.

36. Yesavage JA, BrinkTL, Rose TL, Lum O, Huang V, Adey M, et al. Development and validation of a geriatric depression screening scale: a preliminary report. J Psychiatr Res. 1982;17(1):37-49. https://doi.org/10.1016/ 0022-3956(82)90033-4 PubMed PMID: 7183759. Epub 1982/01/01.

37. Cid-Ruzafa J, Damián-Moreno J. Valoración de la discapacidad física: el indice de Barthel. Rev Esp Salud Pública. 1997;71:127-37.

38. Cabrera González D, Menéndez Caicoya A, Fernández Sánchez A, Acebal García V, García González JV, Díaz Palacios E, et al. Evaluación de la fiabilidad y validez de una escala de valoración social en el anciano. Aten Primaria. 1999;23(7):434-40.

39. Guigoz Y, Vellas B, Garry PJ. Assessing the nutritional status of the elderly: the Mini nutritional assessment as part of the geriatric evaluation. Nutr Rev. 2009;54(1):S59-65. https://doi.org/10.1111/j.1753-4887.1996.tb037 93.x PubMed PMID: 8919685. Epub 1996/01/01.

40. Good M-JD, et al. The family APGAR index: a study of construct validity. J Fam Pract. 1979;8(3):577-82.

41. Gálvez-Cano M, Chávez-Jimeno H, Aliaga-Diaz E. Usefulness of the comprehensive geriatric assessment for evaluating the health of older adults. Revista peruana de medicina experimental y salud publica. 2016;33(2):321-7 Epub 2016/09/24. PubMed PMID: 27656933.

42. Fonseca Alves DJ, Bartholomeu-Neto J, Júnior ER, Ribeiro Zarricueta BS, Nóbrega OT, Córdova C. Walking speed, risk factors, and cardiovascular events in older adults - systematic review. J Strength Cond Res. 2017;31(11):3235-44. https://doi.org/10.1519/jsc.0000000000002182 PubMed PMID: 29065080. Epub 2017/10/25.

43. Viccaro LJ, Perera S, Studenski SA. Is Timed Up and Go Better Than Gait Speed in Predicting Health, Function, and Falls in Older Adults? 
J Am Geriatr Soc. 2011;59(5):887-92. https://doi.org/10.1111/j.15325415.2011.03336.x PubMed PMID: 21410448; PubMed Central PMCID: PMCPMC3522463. Epub 2011/03/18

44. Ferrucci L, Bandinelli S, Benvenuti E, Di lorio A, Macchi C, Harris TB, et al. Subsystems contributing to the decline in ability to walk: bridging the gap between epidemiology and geriatric practice in the InCHIANTI study. J Am Geriatr Soc. 2000;48(12):1618-25. https://doi.org/10.1111/j.15325415.2000.tb03873.x PubMed PMID: 11129752. Epub 2000/12/29.

45. Bohannon RW. Reference Values for the Timed Up and Go Test. J Geriatric Phys Ther (2001). 2006;29(2):64-8. https://doi.org/10.1519/00139143200608000-00004 PubMed PMID: 16914068. Epub 2006/08/18.

46. Studenski S. Bradypedia: is gait speed ready for clinical use? J Nutr Health Aging. 2010;13(10):878-80. https://doi.org/10.1007/s12603-009-0245-0 PubMed PMID: 19924347. Epub 2009/11/20.

47. De Buyser SL, Petrovic M, Taes YE, Toye KRC, Kaufman J-M, Goemaere S. Physical function measurements predict mortality in ambulatory older men. Eur J Clin Investig. 2013;43(4):379-86. https://doi.org/10.1111/eci. 12056 PubMed PMID: 23398295. Epub 2013/02/13.

48. Wang H, Naghavi M, Allen C, Barber RM, Bhutta ZA, Carter A, et al. Global, regional, and national life expectancy, all-cause mortality, and cause-specific mortality for 249 causes of death, 1980-2015: a systematic analysis for the Global Burden of Disease Study 2015. Lancet. 2016;388(10053):1459-544. https://doi.org/10.1016/S0140-6736(16) 31012-1.

49. Mohamed O, Appling H. Clinical assessment of gait. In: Chui KK, Jorge MM, Yen S-C, Lusardi MM, editors. Orthotics and prosthetics in rehabilitation. St. Louis (MO): Elsevier; 2020. p. 102-43.

50. Jung H-W, Kim S, Jang I-Y, Shin DW, Lee JE, Won CW. Screening Value of Timed Up and Go Test for Frailty and Low Physical Performance in Korean Older Population: The Korean Frailty and Aging Cohort Study (KFACS). Ann Geriatric Med Res. 2020;24(4):259-66. https://doi.org/10. 4235/agmr.20.0072 PubMed PMID: 33296961; PubMed Central PMCID: PMCPMC7781966. Epub 2020/12/10

51. Veronese N, Stubbs B, Volpato S, Zuliani G, Maggi S, Cesari M, et al. Association Between Gait Speed With Mortality, Cardiovascular Disease and Cancer: A Systematic Review and Meta-analysis of Prospective Cohort Studies. J Am Med Dir Assoc. 2018;19(11):981-8. e7. Epub 2018/07/30. PubMed PMID: 30056008. https://doi.org/10.1016/j.jamda.2018.06.007.

52. Zhao W, Ukawa S, Tsushita K, Kawamura T, Wakai K, Ando M, et al. Association of gait speed with mortality among the Japanese elderly in the new integrated suburban seniority investigation project: a prospective cohort study. Age Ageing. 2014;44(1):153-7. https://doi.org/10.1093/ageing/ afu121 PubMed PMID: 25236846. Epub 2014/09/23.

53. Galassi A, Reynolds K, He J. Metabolic syndrome and risk of cardiovascular disease: a Meta-analysis. Am J Med. 2006;1 19(10):812-9. https://doi.org/ 10.1016/j.amjmed.2006.02.031.

\section{Publisher's Note}

Springer Nature remains neutral with regard to jurisdictional claims in published maps and institutional affiliations.

Ready to submit your research? Choose BMC and benefit from:

- fast, convenient online submission

- thorough peer review by experienced researchers in your field

- rapid publication on acceptance

- support for research data, including large and complex data types

- gold Open Access which fosters wider collaboration and increased citations

- maximum visibility for your research: over $100 \mathrm{M}$ website views per year

At BMC, research is always in progress.

Learn more biomedcentral.com/submissions 